\title{
Editorial for MobiCASE 2011 Special Issue
}

\author{
Joy Zhang • Jennifer L. Wong
}

Published online: 29 December 2012

(C) Springer Science+Business Media New York 2012

\section{Editorial:}

This special issue features five selected papers with high quality from the Third International Conference on Mobile Computing, Applications and Services (MobiCASE 2011), which was held in Los Angeles, CA, USA, October 24-27, 2011. The first article, "Cross-Compiling Android Applications to iOS and Windows Phone 7", co-authored by A. Puder and O. Antebi, considers a cross compilation approach, whereby Android applications are cross-compiled to $\mathrm{C}$ for iOS and to C\# for Windows Phone 7. This paper describes different aspects of the cross-compiler, from byte code level cross-compilation to API mapping. A prototype of this work (XMLVM) is available under an Open Source license.

The second article titled "Mobile Decentralized Search and Retrieval Using SMS and HTTP" from I. M. Lombera, L. E. Moser, P. M. Melliar-Smith and Y-T. Chuang, describes a decentralized search and retrieval system, named iTrust, that provides resistance against the vulnerabilities of centralized services. The SMS interface and the iTrust SMSHTTP bridge enable SMS-capable mobile phones to communicate with and obtain information from HTTP nodes in the iTrust network. iTrust over SMS protocol enables mobile phones to communicate directly with each other and share information in a peer-to-peer fashion bypassing the

\section{J. Zhang $(\bowtie)$}

Silicon Valley Campus, Carnegie Mellon University,

Pittsburgh, PA, USA

e-mail: joy.zhang@sv.cmu.edu

\section{J. L. Wong}

Department of Computer Science, Stony Brook University, Stony Brook, NY, USA
Internet. This paper also describes the Android user interface, which builds on the basic SMS capabilities of mobile phones and offers a user-friendly way of accessing the iTrust with SMS or iTrust over SMS implementation.

In "Mobile Networks and Applications: A Mobile App Search Engine" the authors investigate a mobile App search engine. The authors (A. Datta, K. Dutta, S. Kajanan and N. Pervin) proposed an independent unbiased search engine Mobilewalla for mobile apps with semantic search capabilities. It has also developed an objective scoring mechanism based on user and developer involvement with an app. The scoring mechanism enables Mobilewalla to provide a number of other ways to discover apps - such as dynamically maintained 'hot' lists and 'fast rising' lists.

The fourth article, "MobiSens: A Versatile Mobile Sensing Platform for Real-world Applications," from P. Wu, J. Zhu and Y. Zhang, discusses a versatile mobile sensing platform for a variety of real-life mobile sensing applications. MobiSens addresses common requirements of mobile sensing applications on power optimization, activity segmentation, recognition and annotation, interaction between mobile client and server, motivating users to provide activity labels with convenience and privacy concerns. Based on the MobiSens platform, authors have developed a range of mobile sensing applications including Mobile Lifelogger, SensCare for assisted living, Ground Reporting for soldiers to share their positions and actions horizontally and vertically, and CMU SenSec, a behavior-driven mobile Security system.

In the last article with the title "Design and Implementation of a Toolkit for Usability Testing of Mobile Apps," the authors (X. Ma, B. Yan, G. Chen, C. Zhang, K. Huang, J. Drury and L. Wang) describes a toolkit that embeds into mobile applications the ability to automatically collect user 
interface (UI) events as the user interacts with the applications. The events are fine-grained and useful for quantified usability analysis. Authors have implemented the toolkit on Android devices and evaluated the toolkit with a real deployed Android application by comparing event analysis (state-machine based) with traditional laboratory testing (expert based). Results show that the proposed toolkit is effective at capturing detailed UI events for accurate usability analysis.

The guest editors are thankful to our reviewers for their effort in reviewing the manuscripts.

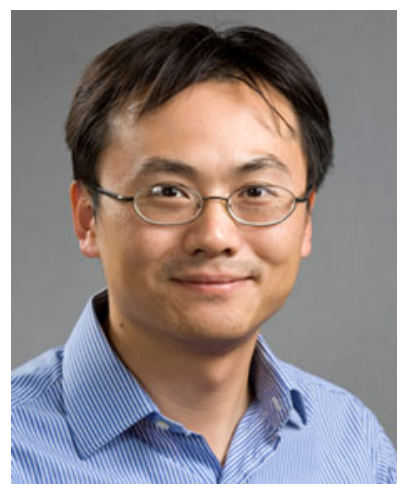

Dr. Joy Zhang is currently a Research Assistant Professor in the Silicon Valley campus of Carnegie Mellon University with courtesy appointments from the CyLab Moblity Research Center, Department of Electrical Computer and Engineering (ECE) and the Language Technologies Institute (LTI). He obtained his BSc from Central South University, China, and MSc degrees from Institute of Computing Technology, Chinese Academy of Sciences, China, and Master of Language Technologies and a $\mathrm{PhD}$ degree from the Carnegie Mellon University, in 1996, 1999, 1991, 2002, and 2008 respectively. He has authored or co-authored over 50 technical papers in major international journals and conferences, books and book chapters in the areas of natural language processing, statistical machine learning, mobile sensing, indoor positioning and behavior modeling. He served as TPC chair, demo/poster chair, TPC member and reviewer for many international conferences and journals.

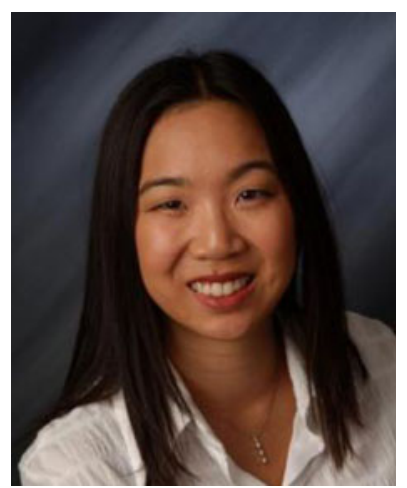

Dr. Jennifer L. Wong is currently an Assistant Professor in the Computer Science Department at Stony Brook University. She obtained her $\mathrm{PhD}$ in 2006 from the Department of Computer Science at the University of California, Los Angeles. Jennifer also has MSc in Computer Science and a BSc in Computer Science and Engineering, also from University of California, Los Angeles. Her research interests include the interaction of statistical models and optimization for computer-aided design of embedded systems and mobile devices, mobile device applications and cloud computing and virtualized environments. Jennifer has served as a reviewer for a variety of IEEE/ACM conferences and journals, and on a number of international conference committees as TPC chair, TPC member, publicity chair and reviewer. 